\title{
Determination of exothermic batch reactor specific model parameters
}

\author{
Lubomír Mackü* \\ Department of Process Control, Faculty of Applied Informatics, Tomas Bata University in Zlin, nám. T. G. Masaryka 5555,76001 Zlín, \\ Czech Republic
}

\begin{abstract}
An alternative method of determining exothermic reactor model parameters which include first order reaction rate constant is described in this paper. The method is based on known in reactor temperature development and is suitable for processes with changing quality of input substances. This method allows us to evaluate the reaction substances composition change and is also capable of the reaction rate constant (parameters of the Arrhenius equation) determination. Method can be used in exothermic batch or semibatch reactors running processes based on the first order reaction. An example of such process is given here and the problem is shown on its mathematical model with the help of simulations.
\end{abstract}

\section{Introduction}

Most of the processes in chemical reactors field usually deals with the same input substances producing the same products all the time. The input substances which are taken from verified suppliers keep their properties, such as a chemical composition, purity, etc., constant. These processes can then be described by mathematical models whose parameters are initially determined for a specific processing procedure and serve for the process control without further changes. There is no reason to change the model parameters unless the initial materials change.

However, from this concept, processes with a previously unknown or only partially known input substances composition are beyond the scope. Examples of a such process may be recycling or regeneration processes [1-3]. The input substances here can be wasted, polluted, or otherwise contaminated raw materials with a major impact on the system's behaviour, and of course also on the system model and/or model parameters. The control algorithm proposed for the original process can then be inappropriate or ineffective. In exothermic reactors area, unexpected uncontrollable temperature rise may occur which in an extreme case might lead even to the reactor destruction [4-5].

One of the important parameters related to exothermic processes is the heat of reaction and other one the variable rate of reaction described by the Arrhenius equation. These parameters can usually be predetermined experimentally and incorporated into the system mathematical model. Unfortunately, in the event of an unexpected change in the composition of the input substances, this procedure is ineffective.

In this work an alternative way of evaluating the feedstock composition changes is described. Here evaluating is based on the reactor response to the addition of the initial test feed put to the reactor. This response can be observed in time temperature profiles development. This response to the initial test feed can serve in "similar" way as response to a unit pulse used in control theory.

This method allows us not only to evaluate the reaction substances composition change, but is also capable of the reaction rate constant (parameters of the Arrhenius equation) determination. Method can be used in exothermic batch or semi-batch reactors running the process based on the first order reaction.

The use of this method will be demonstrated on the real example of an exothermic semi-batch reactor used for the chrome leather waste recycling technology.

\section{Semi-batch exothermic reactor model}

The following figure (figure 1) shows a chemical semi-batch reactor with initial filling $m_{B}[\mathrm{~kg}]$ given by the solution of chemicals. A reactant causing an exothermic chemical reaction to run is fed into the reactor to control the developing heat. The temperature has to stay under a certain critical level; otherwise the reactor could be destroyed. It is also desirable to utilize the whole capacity of the reactor to process the maximum amount of chemicals in the shortest possible time (higher temperature is desirable). The reactor has a double wall for cooling medium and we consider that its content is ideally stirred. We can consider this chemical exothermic semi-batch reactor as a single input - single output (SISO) system from the perspective of automatic control.

\footnotetext{
Corresponding author: macku@utb.cz
} 


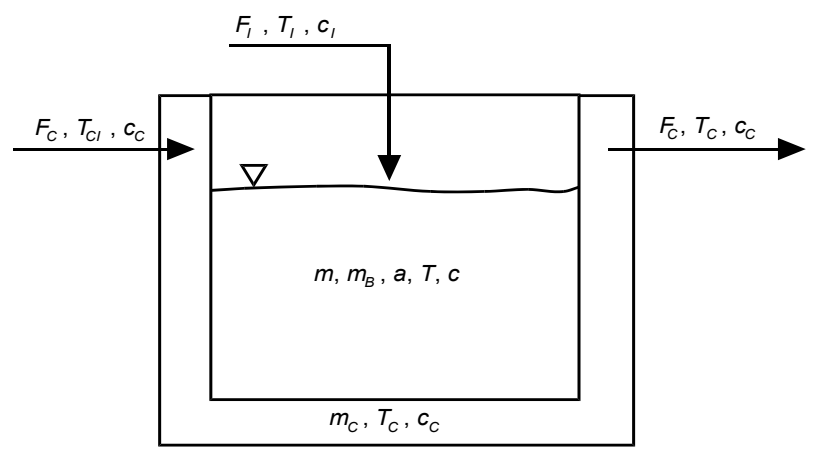

Fig. 1. Exothermic semi batch reactor scheme

The mathematical model of such system can be written by equations (1)-(4):

$$
\begin{gathered}
\frac{\mathrm{d} m(t)}{\mathrm{d} t}=F_{I} \\
\frac{\mathrm{d} a(t)}{\mathrm{d} t}=\frac{F_{I}[1-a(t)]}{m(t)}-A \cdot e^{-\frac{E}{R \cdot T(t)}} \cdot a(t) \\
\frac{\mathrm{d} T(t)}{\mathrm{d} t}=\frac{F_{I} \cdot c_{I} \cdot T_{I}}{m(t) \cdot c}+\frac{A \cdot e^{-\frac{E}{R \cdot T(t)}} \cdot \Delta H_{r} \cdot a(t)}{c}- \\
\frac{\mathrm{d} T_{C}(t)}{\mathrm{d} t}=\frac{F_{C} \cdot S \cdot T(t)}{m(t) \cdot c}+\frac{K \cdot S \cdot T_{C}(t)}{m(t) \cdot c}-\frac{T(t) F_{I}}{m(t)} \\
m_{C}+\frac{K \cdot S(t)}{m_{C} \cdot c_{C}}- \\
-\frac{K \cdot S \cdot T_{C}(t)}{m_{C} \cdot c_{C}}-\frac{F_{C} \cdot T_{C}(t)}{m_{C}}
\end{gathered}
$$

The above described mathematical model was set up for the chromium waste recycling process [6-7]. Here $m$ is the total weight of reaction components in the reactor, $a$ is the mass concentration of the reaction component in the reactor, $c$ is the specific heat capacity of the reactor content; $T$ is the temperature of the reactor content. $F_{I}, T_{I}$ and $c_{I}$ are the reaction component input mass flow rate, temperature and specific heat capacity. $F_{C}, T_{C I}, T_{C}, c_{C}$ and $m_{C}$ are the cooling water mass flow rate, input temperature, output temperature, specific heat capacity and weight of the cooling water in the cooling system of the reactor, respectively. Other constants: $A$ preexponential factor, $E$ activation energy, $R$ gas constant, $\Delta H r$ heat of reaction, $K$ heat transfer coefficient, $S$ heat transfer surface.

\subsection{The rate constant}

Equations (2) and (3) include the temperature and the reaction component mass concentration influence on the reaction rate. The temperature dependence is defined by Arrhenius equation (5), were $k$ is the rate constant:

$$
k=A e^{-\frac{E}{R T[t]}}
$$

The rate constant causes an accumulation of the reaction component in the reactor without corresponding temperature rise. Then, the level of concentration which starts the reaction is reached and the reaction runs very fast. The temperature rises as well and causes the reaction runs even faster. Thus, if the amount of nonreacted reaction component in the reactor is too high, the temperature became uncontrollable and we can't avoid its overshooting. An example of the temperature overshoot caused by accumulation can be seen in figure 2. Here you can see a temperature overshoot over $373 \mathrm{~K}$, which was caused by the accumulation of the reaction component. The corresponding mass concentration is depicted in the figure 3 . The plots were obtained by two step control simulation. The actuating signal switch-off was set on $370 \mathrm{~K}$, the switch-on was set on $365 \mathrm{~K}$. It was impossible prevent the temperature overshooting and simultaneously to trace the desired value $373 \mathrm{~K}$. In the steady state (from approximately $\mathrm{t}=2000 \mathrm{~s}$ ) was the control performance satisfactory. So, it is necessary to take into account the mass concentration to prevent the temperature overshooting.

The in-reactor temperature

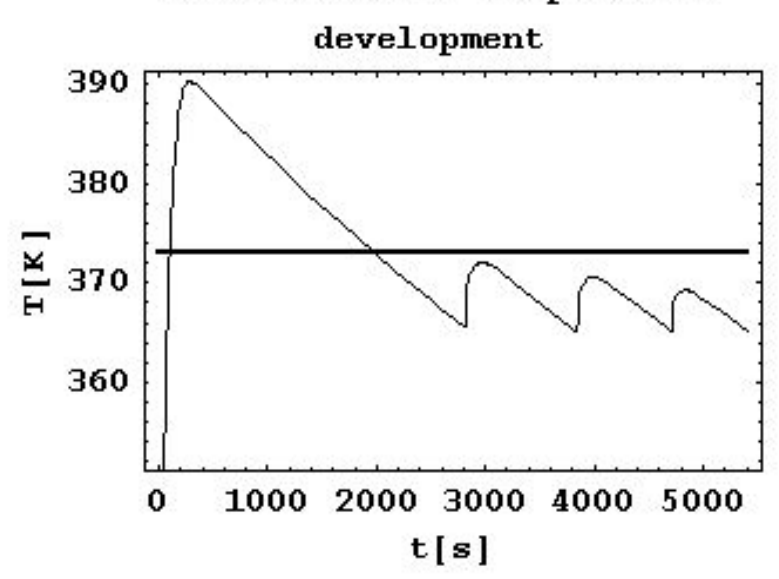

Fig. 2 Overshoot caused by the reactant accumulation

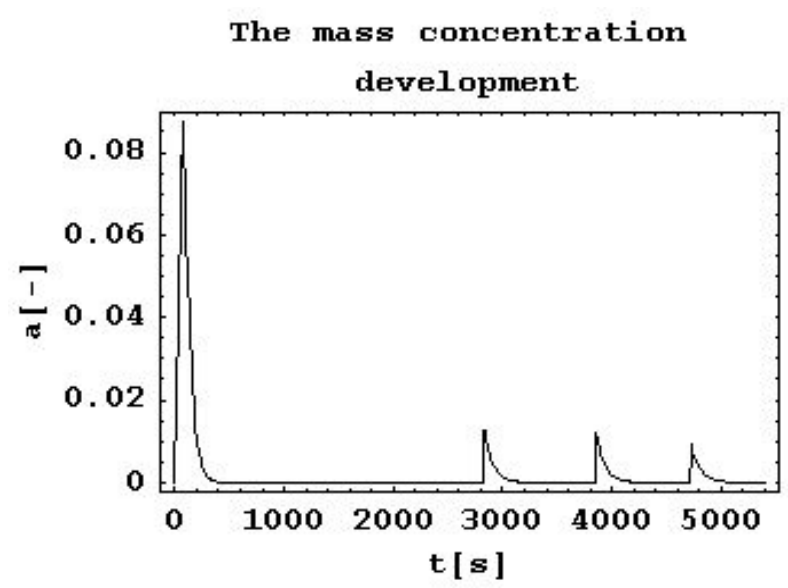

Fig. 3 Mass concentration development (no penalization) 


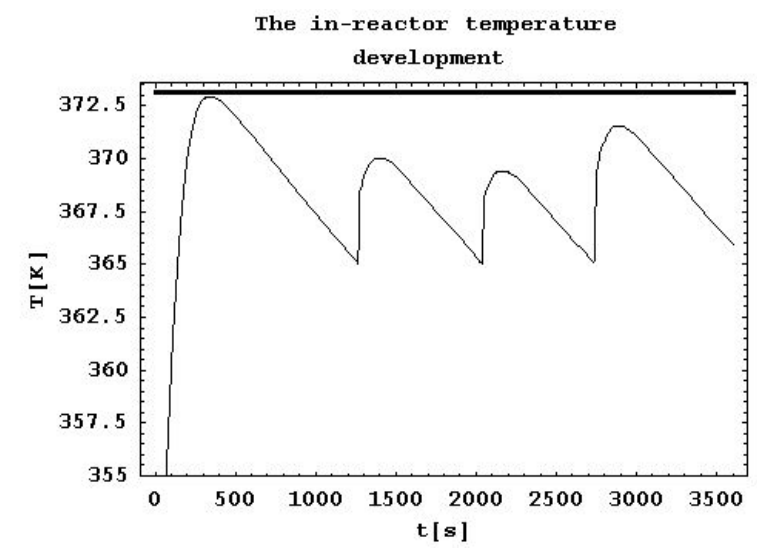

Fig. 4 Temperature development (penalized values)

With the knowledge of the previously mentioned model (that means also the knowledge of the rate constant) we can find the safe value of the reaction component concentration during the start-up part of the process control and so prevent the temperature overshooting as can be seen in figure 4 . The corresponding mass concentration development is than showed in the figure 5 .

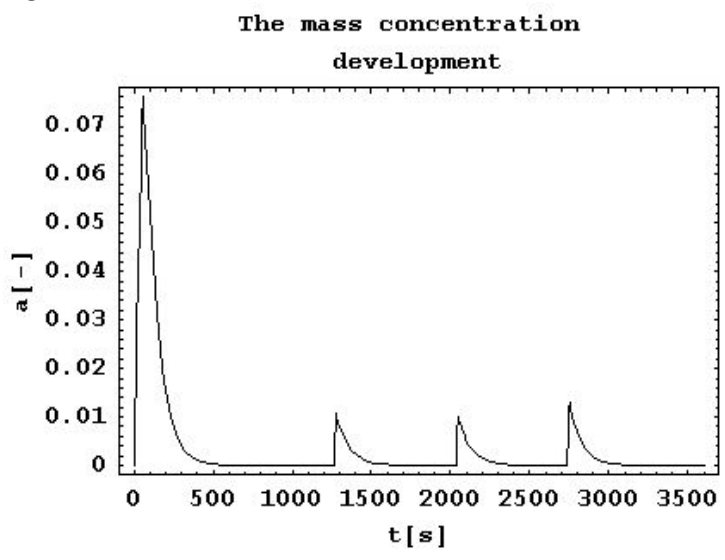

Fig. 5 The mass concentration development (penalized values)

Plots show simulation of two-step control with the temperature and reaction component penalization. In case the reaction component mass concentration reaches the set value and the temperature gets over penalized value at the same time the actuating variable $F_{I}$ is switched off.

The non-reacted chemical reactant is so prevented from cumulating in the chemical reactor. The insensitivity zone was chosen at interval $\left(T_{P P}+1>T[t] \geq T_{P P}\right)$, to prevent the in-reactor temperature to oscillate around the penalty temperature value.

As can be seen from the above mentioned text, it is important to know the velocity constant parameters to be able satisfactory control like these processes. As far as we use the very same chemicals for processing, there is generally no problem. We can do the identification once and the parameters are always the same. But in case we plan wider range of chemicals processing, or the mixture properties are changeable in time, it is necessary to perform an on-line velocity coefficient identification to process different mixture types safely.

\section{Rate constant identification}

In this chapter the procedure for the Arrhenius equation parameters identification is described.

The heat $Q$ can be expressed by equation:

$$
Q=C \Delta T_{1}
$$

where $C$ is the system heat capacity (the heat needed for the system warming up for $1 \mathrm{~K}$ ) and $\Delta T_{1}$ the system temperature increase caused by the generated heat.

As was mentioned before, we assume that the exothermic chemical reaction can be treated as a first order reaction from the reaction kinetic point of view. That means that the reaction velocity $v$ is directly proportional only to one of the reactive components concentration $c$, as can be seen in formula (7).

$$
v=-d c / d t=k c
$$

Here the symbols have the following meaning: $v$ - reaction velocity, $c$-concentration of the reaction component, $t$ - time, $k$ - velocity constant. The velocity constant $k$ can be generally determined by measurement of any property which is proportional to the concentration. Since we are interested in exothermic reaction, it seems to be possible to trace the amount of heat generated by the chemical reaction. This generated heat is adequate to number of reacted moles and so also to the concentration of the reaction component.

The equation (6) implies that the system temperature increase corresponds to the generated heat as can be seen from the equation (8) below:

$$
d T=\frac{d Q}{C}
$$

We also know, that the increase of heat $d Q$ released by the chemical reaction matches the product of reacted moles $d n$ and the heat of reaction $\Delta H_{r}$. It can be written:

$$
d Q=\Delta H_{r} d n=d c V \Delta H_{r}
$$

where the product of concentration changes and the volume of reaction mixture matches the number of moles. After dividing the equation (8) by time $t$ and with the help of substituting equation (9) we obtain the relationship (10):

$$
\frac{d T}{d t}=\frac{V \Delta H_{r}}{C} \frac{d c}{d t}
$$

where the term $d c / d t$ expresses the reaction rate, see relation (7). Putting (7) into (10) allows us to extrapolate the temperature derivative at zero time. We obtain the relationship: 


$$
v=-\frac{d T}{d t} \frac{C}{V \Delta H_{r}}
$$

By comparing relations (11) with relation (7) we get the equation for rate constant:

$$
k=\frac{d T}{d t} \frac{C}{V \Delta H_{r} c}=\frac{d T}{d t} \frac{C}{\Delta H_{r} n}
$$

Individual symbols meaning: derivation $d T / d t$ means a tangent direction (see figure 6), $C\left[\mathrm{~J}_{\mathrm{K}} \mathrm{K}^{-1}\right]$ - system thermal capacity, $V\left[\mathrm{~m}^{3}\right]$ - volume, $c\left[\mathrm{~mol} . \mathrm{m}^{-3}\right]$ - molar concentration, $n[\mathrm{~mol}]$ - the number of mole.

As can be seen from the formula (12) we need to know the in-reactor chemicals volume $V$ and concentration of reactant causing the heat development $c$ (or the number of mole $n$ instead) to determine the rate constant. Further it is necessary to know also the heat of reaction $\Delta H r$, system thermal capacity $C$ and the temperature tangent direction $d T / d t$.

By substituting the experimentally observed data to the expression (12) we can calculate its value - the rate constant for the different initial reaction temperatures. The derivative $d T / d t$ is actually the tangent to the curve of $T$ dependence as a function of $t$. The tangent value can be determined from the temperature-time graph, which is obtained by recording the temperature during a chemical reaction, see figure 6 .

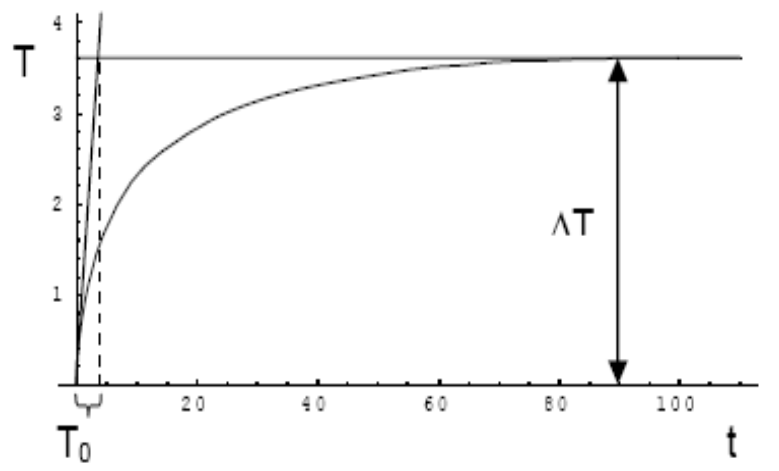

Fig. 6 An example of reaction temperature time dependence

It is advantageous to read the directive in the start of the reaction, because we know the initial concentrations of substances. Therefore, the known initial concentration $c_{0}$ can be put into relation (12) for the concentration $c$. The volume $V$ of reaction mixture is also known and the value of reaction heat $\Delta H_{r}$ can be found experimentally.

To determine the rate constant temperature dependence, we use the Arrhenius relationship in the shape of a logarithmic form (13):

$$
\ln k=\ln A-\frac{E}{R T}
$$

Plotting the dependence $\ln k$ on $1 / T$ gives a linear dependence in the form $y=a+b x$. From this dependence we find values $a$ and $b$. By comparing this dependence with the relation (13) we obtain relationships for calculating pre-exponential factor $A$ (14) and activation energy $E$ (15):

$$
\begin{gathered}
A=e^{a} \\
E=-b R
\end{gathered}
$$

These constants are used to compile the model equations of the chemical reactor.

The chemicals volume and the starting concentration are usually known. The system (reactor) thermal capacity must be determined, but only once. Finally, the temperature tangent direction may be obtained from the measured temperature time dependence. With the help of the formula (6) we can use an in-reactor temperature profile to find the rate constant directly from the process (in case we put known chemicals quantity in the reactor). The whole procedure had been already verified by author during the chromium sludge processing [8].

\section{Conclusion}

The semi-batch and batch exothermic reactor control is a complex and difficult process. One of the difficulties is the changing reaction rate which is causing the system nonlinearity as was shown in this paper. However, it is possible to deal with the problem by the rate constant identification based on the temperature profiles.

An alternative method of determining exothermic reactor model parameters suitable for first order reactions was presented in this paper. With the knowledge of the in reactor temperature development the reaction substances composition and the reaction rate constant (parameters of the Arrhenius equation) change can be evaluated. In case we put known chemicals quantity in the reactor the rate constant can be found directly from the controlled process. We need to know the inserted chemicals volume and their starting concentrations, which are usually known. The system (reactor) thermal capacity must be determined, but for first time only. The temperature tangent direction may be obtained from the measured temperature time dependence, also the reaction heat could be found from the same.

The method is usable especially for processes with changing quality of input substances occurring in exothermic batch or semi-batch reactors. An example of such process was given here and the problem was shown on the reactor mathematical model using the process simulations.

The rate constant knowledge can help us to set up a precise system model and to apply a suitable control method. With a system model even simple control methods can be used to control some specific batch reactors. On the above mentioned system some control methods had been applied, which were described in articles [9-11]. Very powerful seems to be for example predictive control using neural networks [9]. 


\section{References}

1. M. Heidari, S. Salaudeen, A. Dutta, B. Acharya, Energy \& Fuels, 32(11), 11576-11586, (2018)

2. F. Maestri, C. Pirola, R. Rota, Chemical Engineering Transactions, 67, 31-36, (2018)

3. S. Schwolow, A. Neumüller, L. Abahmane, N. Kockmann, T. Röder, Chemical Engineering and Processing: Process Intensification, 108, 109-116, (2016)

4. A. Kummer, T. Varga Chemical Engineering Science, 196, 277-290, (2019)

5. J. Cui, L. Ni, J. Jiang, Y. Pan, H. Wu, Q. Chen Organic Process Research \& Development, 23(3), 389-396, (2019)

6. K. Kolomazník, M. Adámek, M. Uhlířová, Proceedings of the 5th IASME/WSEAS International Conference on Heat Transfer, Thermal Engineering and Environment, 137-141, (2007)

7. L. Macků, D. Sámek, WSEAS Transactions on Systems, 9(1), 1039-1049, (2010)

8. L Macků, Ph.D. Thesis, UTB in Zlin, (2003)

9. L. Macků, D. Sámek, Computer Science On-line Conference ,255-265, (Springer 2016)

10. F. Gazdoš, L. Macků, Proceedings of 22nd European Conference on Modelling and Simulation ECMS 2008, 512-518, (2008)

11. D. Novosad, L. Macku, Annals of DAAAM for 2010 \& Proceedings of the 21st International DAAAM Symposium, 1017-1018, (2010) 